\title{
The Knee injury and Osteoarthritis Outcome Score reflects the severity of knee osteoarthritis better than the revised Knee Society Score in a general Japanese population
}

\author{
Kazuki Oishi a,*, Eiichi Tsuda a , Yuji Yamamoto a , Shugo Maeda ${ }^{\text {a }}$, Eiji Sasaki ${ }^{\text {a }}$, Daisuke Chiba ${ }^{\text {a }}$, Ippei Takahashi ${ }^{\text {b }}$ \\ Shigeyuki Nakaji ${ }^{\mathrm{b}}$, Yasuyuki Ishibashi ${ }^{\mathrm{a}}$ \\ a Department of Orthopedic Surgery, Hirosaki University Graduate School of Medicine, Hirosaki, Japan \\ b Department of Social Medicine, Hirosaki University Graduate School of Medicine, Hirosaki, Japan
}

\section{A R T I C L E I N F O}

\section{Article history:}

Received 1 May 2015

Received in revised form 29 July 2015

Accepted 10 August 2015

Available online $\mathrm{xxxx}$

\section{Keywords:}

Knee Society Score

Knee injury and Osteoarthritis Outcome Score

Knee osteoarthritis

General population

\begin{abstract}
A B S T R A C T
Purpose: The purposes of this study were to examine population-based reference data for sex- and age-related differences between the 2011 revised Knee Society Score (KSS2011) and the Knee injury and Osteoarthritis Outcome Score (KOOS), to assess the correlation between those scores and radiographic knee osteoarthritis (OA), and to validate the use of the scores in a general Japanese population.

Methods: This cross-sectional study included 963 volunteers ( 368 males, 595 females; mean age: 54.7 years). Participants were classified into five subgroups by age: under 40, 40s, 50s, 60s, and over 70 years old. The KSS2011 and KOOS were determined using self-administered questionnaires. Weight-bearing radiographs of the bilateral knee were taken and graded according to the Kellgren-Lawrence (KL) scale. The mean KSS2011 and KOOS were compared among age groups. Correlations between the severity of knee OA and each score were assessed using multiple regression analysis.

Results: The overall KSS2011 tended to gradually decrease with age. Most subscales of the KSS2011 did not show sex-related differences. Similarly, the overall KOOS and all its subscales steadily decreased by approximately 20 points per decade with age. Most subscales of the KOOS were significantly decreased in females over 50 . The KL grade was significantly related to both the overall KOOS $(\beta=-0.42, \mathrm{p}<0.001)$ and $\mathrm{KSS} 2011(\beta=-0.13$, $\mathrm{p}=0.001)$, though the correlation to the KOOS was stronger.

Conclusion: The overall KSS2011 and KOOS appear to decrease with age. In this population, the KOOS reflects the severity of knee OA better than the KSS2011.
\end{abstract}

(C) 2015 Elsevier B.V. All rights reserved.

\section{Introduction}

Knee osteoarthritis (OA) is a common disorder that causes pain and disability to an increasing fraction of the population. Various risk factors associated with the incidence and progression of OA have been identified, including aging, high body mass index (BMI), female sex, previous knee injury, quadriceps muscle strength, and knee malalignment, among others [1-4]. Of these, obesity and quadriceps muscle strength are important for the prevention and suppression of the disease. To relieve knee pain and improve knee function, total knee arthroplasty (TKA) is one of the treatment options for cases of severe knee $\mathrm{OA}$. Over the last two decades, the number of younger and more active patients undergoing TKA has increased [5]. Traditionally, the surgical outcomes after knee surgery have been evaluated objectively using radiographic imaging and the surgeon's assessment of the joint functions,

* Corresponding author at: Department of Orthopaedic Surgery, Hirosaki University Graduate School of Medicine, 5 Zaifu-cho, Hirosaki, Aomori 036-8562, Japan. Tel: +81 17239 5083; fax: +81172363826 including the stability, lower limb alignment, and range of motion. To better characterize functional activity in line with the demands of younger patients, the traditional clinical scales have been adapted and new scales have been developed. Furthermore, patient-reported outcome scales also play an important role in the assessment of patients with knee problems after injury or in OA [6].

The Knee Society Knee Scoring System (KSS) was developed to evaluate the function of both knee prostheses and a patient's functional abilities after TKA [7]. This original score was based on only physicianderived variables, leaving an unresolved poor correlation between objective physician-assessed knee scores and patient-derived satisfaction scores. In 2011, the KSS was revised (KSS2011) to better characterize the expectations, satisfaction, and physical activities of a more diverse population of patients [8]. The KSS2011, a questionnaire that includes subjective and objective evaluations, has enabled the comprehensive assessment of patient knee function before and after surgery with high reliability $[9,10]$

The validation of the KSS2011 was performed in patients with knee OA before and after TKA [11]. The patient satisfaction, expectation, and 
Table 1

Number, age, BMI, and radiographic knee OA of the study participants by age group.

\begin{tabular}{|c|c|c|c|c|c|c|c|c|c|c|}
\hline & \multicolumn{2}{|l|}{$<40$} & \multicolumn{2}{|l|}{$40 \mathrm{~s}$} & \multicolumn{2}{|l|}{$50 \mathrm{~s}$} & \multicolumn{2}{|l|}{$60 \mathrm{~s}$} & \multicolumn{2}{|l|}{$\geq 70$} \\
\hline & M & $\mathrm{F}$ & M & $\mathrm{F}$ & M & $\mathrm{F}$ & M & $\mathrm{F}$ & M & $\mathrm{F}$ \\
\hline Participants number & 92 & 113 & 55 & 86 & 62 & 130 & 96 & 166 & 63 & 100 \\
\hline \multirow[t]{2}{*}{ Age (years) } & 32.1 & 33.1 & 45.0 & 45.0 & 54.4 & 55.5 & 64.0 & 64.2 & 75.2 & 75.0 \\
\hline & 31.1 to 33.2 & 32.2 to 34.0 & 44.3 to 45.7 & 44.3 to 45.6 & 53.7 to 55.1 & 55.0 to 56.0 & 63.4 to 64.5 & 63.8 to 64.6 & 74.1 to 76.4 & 74.2 to 75.9 \\
\hline \multirow[t]{2}{*}{ BMI $\left(\mathrm{kg} / \mathrm{m}^{2}\right)$} & 23.2 & 21.0 & 24.0 & 22.2 & 23.5 & 22.1 & 23.3 & 23.1 & 23.4 & 22.7 \\
\hline & 22.4 to 23.9 & 20.3 to 21.7 & 23.2 to 24.7 & 21.5 to 22.9 & 22.8 to 24.2 & 21.6 to 22.7 & 22.7 to 23.9 & 22.6 to 23.7 & 22.6 to 24.2 & 22.1 to 23.3 \\
\hline $\begin{array}{l}\text { Number of participants with } \\
\text { radiographic knee OA (\%) }\end{array}$ & $3(3.3)$ & $12(10.6)$ & $7(12.7)$ & $22(25.6)$ & $16(25.8)$ & $68(52.3)$ & $37(38.5)$ & $113(68.1)$ & $35(55.6)$ & $84(84.0)$ \\
\hline
\end{tabular}

Values of age and BMI are given as the mean and 95\% confidence interval.

BMI: body mass index.

functional activities subscales of the KSS2011 questionnaire were correlated with each subscale of the Short Form-12 (SF-12) score and the Knee injury and Osteoarthritis Outcomes Score (KOOS) [11]. The Japanese version of the KOOS has been validated [12] and is reported to be a useful tool for evaluating the knee condition after TKA [13]. The Japanese version of the KSS2011 has also been used to evaluate patient satisfaction and daily activities after TKA [14]. Based on a study of the general Japanese population, Taniguchi et al. reported that the KSS2011 decreased with age and was correlated with radiographic knee OA in people older than 60 years. However, changes in the KSS2011 by sex and age group in comparison with other objective rating systems and the correlation with severity of knee OA by age group have not yet been reported. The purposes of this large-scale crosssectional study were to examine the changes in the KSS2011 by sex and age group compared with the KOOS and to assess the correlations between these scores and the severity of knee $\mathrm{OA}$ in a general Japanese population.

\section{Materials and methods}

\subsection{Subjects}

The subjects in this study were volunteers who participated in the Iwaki Health Promotion project. This annual project, running since 2005 , is a community-based program with the goal of improving the average life expectancy by performing general health checkups on the general population living in the Iwaki area of Hirosaki city, which is located in western Aomori prefecture, Japan [15,16]. Informed consent was obtained from all individual participants included in the study, and the protocols used in this study were conducted with the approval of the ethics committee of Hirosaki University School of Medicine. A total of 1045 volunteers from among the 12,000 residents participated in the Iwaki Health Promotion project in 2013. Of these, 23 patients with prior knee surgery, 28 participants who did not receive

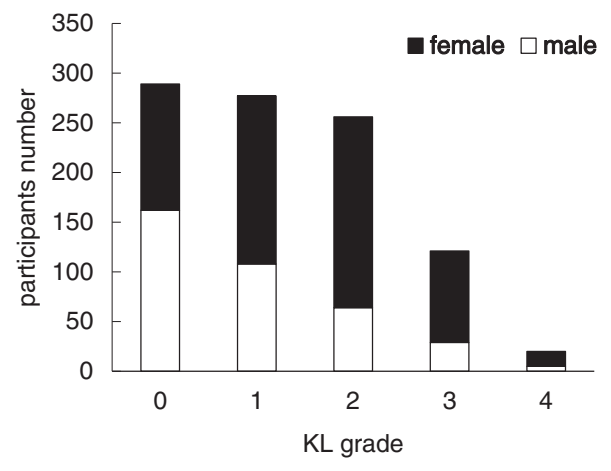

Fig. 1. Distribution of the Kellgren-Lawrence (KL) grades in the participants. Most of the participants who had knee OA were female with grade 2. radiography, and 31 participants who did not complete the questionnaire were excluded from this study, leaving 963 participants who were assessed in the present study. The KSS2011 and KOOS questionnaires for knee condition and radiographs of the knee were taken for these participants.

\subsection{Evaluation of knee osteoarthritis}

Bilateral weight-bearing and anterior-posterior radiographs of the knees were taken. All of the knee radiographs were graded by two trained orthopedic surgeons blinded to the participant data. The severity of each knee was graded based on the Kellgren-Lawrence (KL) grade [17]. The presence of radiographic knee OA was defined as a KL grade of 2,3 , or 4 . The participants were classified as either OA or non-OA using their worse knee score.

\subsection{Revised Knee Society Score (KSS2011)}

Each participant filled out the self-administered areas of the KSS2011 questionnaire, including "symptoms" (three items; 25 points), "patient satisfaction" (five items; 40 points), and "functional activities" (19 items: 100 points). The "symptoms" section consisted of two numerical rating scales completed by the participants and one question about the frequency of the abnormality. The "patient satisfaction" section consisted of the pain level while sitting or lying in bed and knee function while getting out of bed, performing light household duties, and performing leisure or recreational activities. The "expectation" sections of the questionnaire were excluded because the participants did not plan to undergo TKA. The "functional activities" consisted of "walking and standing", "standard activities" (standing from a seated position and going up and down stairs), "advanced activities" (squatting down deeper and going up a ladder or running), and "discretionary activities" (18 sports activities listed). The score for each subscale was calculated by summing the points for each item listed and was considered independent from the other domains. Higher scores indicate better outcomes in all of the subscales. The discretionary activities score was excluded from further analysis because the response rate for that section was low. The maximum possible score from these questions was 150 points.

\subsection{Knee injury and Osteoarthritis Outcome Score (KOOS)}

The KOOS is based on 42 knee-related items each scored from 0 to four. Five patient-relevant categories: pain (nine items), other disease-specific symptoms (symptoms; seven items), function in activities of daily living (ADL; 17 items), sport and recreation function (sports/recreation; five items), and knee-related quality of life (QOL; four items) were converted to a 100 point scale [18]. The KOOS items were scored from 0 to four and summed within each subscale. These scores were converted to percentage scores with higher scores 


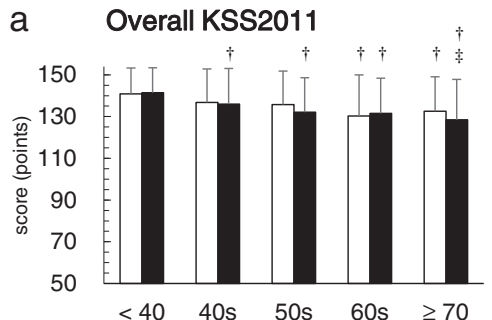

C

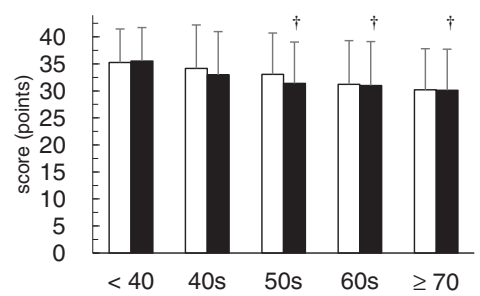

e b Symptoms

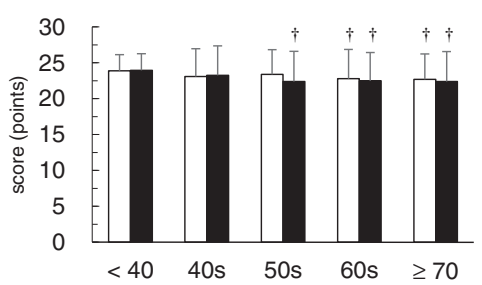

d

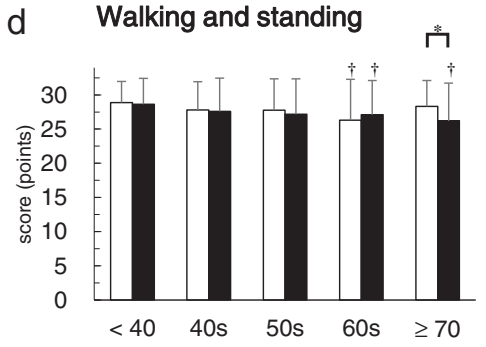

f Advanced activities

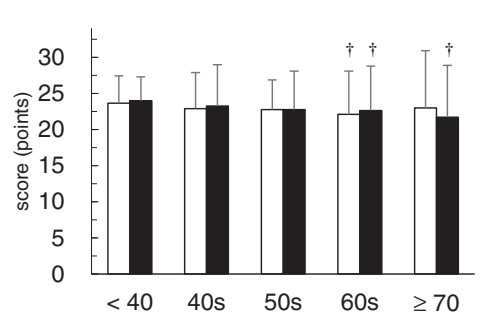

male female

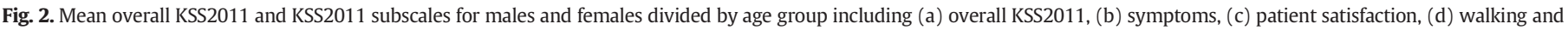

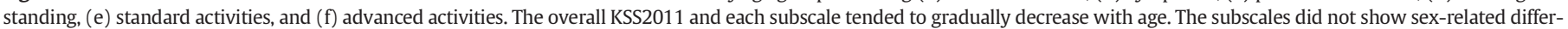

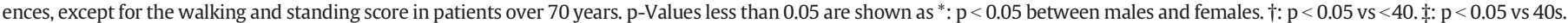
$\S: \mathrm{p}<0.05$ vs 50 s. $\|: \mathrm{p}<0.05$ vs 60 s. Overall KSS: the total KSS2011 summed from the individual subscales.

Table 2

KSS2011 and KOOS reported as mean and 95\% confidence interval for males (M) and females (F) by age group.

\begin{tabular}{|c|c|c|c|c|c|c|c|c|c|c|}
\hline & \multicolumn{2}{|l|}{$<40$} & \multicolumn{2}{|l|}{$40 \mathrm{~s}$} & \multicolumn{2}{|l|}{$50 \mathrm{~s}$} & \multicolumn{2}{|l|}{$60 \mathrm{~s}$} & \multicolumn{2}{|l|}{$\geq 70$} \\
\hline & M & $\mathrm{F}$ & M & $\mathrm{F}$ & M & $\mathrm{F}$ & M & $\mathrm{F}$ & M & $\mathrm{F}$ \\
\hline \multirow[t]{3}{*}{ Overall KSS2011 } & 140.9 & 141.5 & 136.7 & $136.0^{*}$ & 135.7 & $132.1^{*}$ & $130.3^{*}$ & $131.5^{*}$ & $132.5^{*}$ & $128.4^{*} \dagger$ \\
\hline & 138.3 to & 139.2 to & 132.4 to & 132.4 to & 131.6 to & 129.2 to & 126.3 to & 128.9 to & 128.3 to & 124.5 to \\
\hline & 143.4 & 143.7 & 141.1 & 139.7 & 139.8 & 135.0 & 134.3 & 134.1 & 136.7 & 132.2 \\
\hline \multirow[t]{2}{*}{ Symptoms } & 23.9 & 24.0 & 23.1 & 23.3 & 23.4 & $22.4^{*}$ & $22.8^{*}$ & $22.5^{*}$ & $22.7^{*}$ & $22.4^{*}$ \\
\hline & 23.4 to 24.3 & 23.5 to 24.4 & 22.0 to 24.1 & 22.4 to 24.1 & 22.5 to 24.2 & 21.6 to 23.1 & 21.9 to 23.6 & 21.9 to 23.1 & 21.9 to 23.6 & 21.6 to 23.2 \\
\hline \multirow[t]{2}{*}{ Patient satisfaction } & 35.3 & 35.5 & 34.2 & 33.0 & 33.1 & $31.4^{*}$ & 31.2 & $31.0^{*}$ & 30.2 & $30.1^{*}$ \\
\hline & 34.3 to 36.6 & 34.4 to 36.7 & 32.1 to 36.3 & 31.2 to 34.7 & 31.1 to 35.0 & 30.0 to 32.7 & 29.6 to 32.8 & 29.7 to 32.2 & 28.1 to 32.3 & 28.6 to 31.7 \\
\hline \multirow{2}{*}{$\begin{array}{l}\text { Walking and } \\
\text { standing }\end{array}$} & 28.9 & 28.6 & 27.8 & 27.6 & 27.8 & 27.2 & $26.3^{*}$ & $27.1^{*}$ & 28.39 & $26.2^{*}$ \\
\hline & 28.3 to 29.5 & 27.9 to 29.3 & 26.7 to 28.9 & 26.5 to 28.6 & 26.6 to 28.9 & 26.3 to 28.1 & 25.0 to 27.5 & 26.3 to 27.9 & 27.4 to 29.3 & 25.1 to 27.3 \\
\hline \multirow[t]{2}{*}{ Standard activities } & 29.2 & 29.3 & 28.8 & 29.0 & 28.8 & 28.4 & 28.0 & $28.4^{*}$ & 28.3 & $28.0^{*}$ \\
\hline & 28.8 to 29.6 & 29.0 to 29.7 & 28.0 to 29.5 & 28.4 to 29.5 & 28.1 to 29.5 & 27.8 to 29.1 & 27.2 to 28.6 & 28.8 to 28.9 & 27.4 to 29.1 & 27.1 to 28.8 \\
\hline \multirow[t]{2}{*}{ Advanced activities } & 23.7 & 24.0 & 22.9 & 23.3 & 22.7 & 22.7 & $22.1^{*}$ & $22.6^{*}$ & 23.0 & $21.7^{*}$ \\
\hline & 23.1 to 24.2 & 23.5 to 24.4 & 21.8 to 24.0 & 22.4 to 24.1 & 21.7 to 23.8 & 22.0 to 23.5 & 21.1 to 23.0 & 21.8 to 23.3 & 21.9 to 24.0 & 20.7 to 22.8 \\
\hline \multirow[t]{2}{*}{ Overall KOOS } & 487.9 & 481.8 & 484.2 & 473.2 & 471.29 & $450.1^{*} \dagger$ & $452.1^{*}+9$ & $431.7^{*} \dagger$ & $449.6^{*}+9$ & $409.1^{*}+$ \\
\hline & 483.2 to 492.7 & 476.1 to 487.6 & 477.2 to 491.3 & 462.7 to 483.8 & 458.2 to 484.3 & 438.7 to 461.5 & 437.5 to 466.7 & 419.6 to 443.8 & 432.4 to 466.9 & 391.0 to 427.1 \\
\hline \multirow[t]{2}{*}{ Pain } & 96.1 & 94.5 & 95.6 & 94.4 & 93.79 & $91.0^{*}+$ & $92.7^{*}+9$ & $89.1^{*}+\frac{1}{7}$ & $92.5 * 9$ & $88.2^{*}+\ddagger$ \\
\hline & 95.0 to 97.2 & 93.2 to 95.9 & 94.1 to 97.2 & 92.4 to 96.4 & 90.9 to 96.4 & 88.9 to 93.3 & 90.1 to 95.3 & 87.0 to 91.3 & 89.5 to 95.5 & 85.3 to 91.2 \\
\hline \multirow[t]{2}{*}{ Symptoms } & 98.7 & 97.1 & 97.5 & 95.9 & 95.49 & 92.0 & 92.09 & $89.2^{*} \dagger$ & 92.99 & $86.1^{*} \dagger$ \\
\hline & 97.9 to 99.5 & 95.8 to 98.4 & 96.0 to 99.0 & 93.9 to 97.9 & 93.1 to 97.8 & 89.9 to 94.1 & 89.2 to 94.7 & 86.9 to 91.4 & 89.7 to 96.0 & 82.7 to 89.6 \\
\hline \multirow[t]{2}{*}{ ADL } & 99.5 & 99.3 & 99.49 & 98.2 & 98.39 & $95.9^{*}$ & $95.6^{*}+9$ & $93.7^{*} \dagger$ & $94.0 *+\frac{1}{T} 9$ & $89.4^{*}+\ddagger$ \\
\hline & 99.1 to 99.9 & 96.7 to 99.3 & 98.7 to 100.2 & 93.6 to 99.0 & 96.9 to 99.7 & 87.6 to 93.8 & 93.6 to 97.6 & 80.6 to 87.8 & 91.2 to 96.7 & 66.7 to 78.6 \\
\hline \multirow[t]{2}{*}{ Sports/recreation } & 97.9 & 98.0 & 98.5 & 96.3 & 95.6 & $90.7^{*}$ & $88.4^{*}+9$ & $84.2 * \dagger$ & $84.6 *+5$ & $72.6^{*}+\ddagger \S$ \\
\hline & 96.6 to 99.2 & 90.8 to 95.2 & 97.2 to 99.9 & 84.6 to 92.2 & 92.3 to 99.0 & 76.8 to 84.1 & 84.1 to 92.6 & 71.9 to 79.3 & 79.3 to 89.9 & 67.7 to 77.5 \\
\hline \multirow[t]{2}{*}{ QOL } & 95.79 & 93.0 & 93.1 & 88.4 & $88.2 * \mathrm{G}$ & $80.5^{*} \dagger$ & $83.5^{*}+9$ & $75.6^{*} \dagger$ & $85.7^{*} \mathrm{~g}$ & $72.6^{*} \dagger$ \\
\hline & 93.7 to 97.8 & 90.8 to 95.2 & 89.5 to 96.7 & 84.6 to 92.2 & 83.6 to 92.6 & 76.8 to 84.1 & 79.0 to 87.9 & 71.9 to 79.3 & 80.5 to 90.9 & 67.7 to 77.5 \\
\hline
\end{tabular}

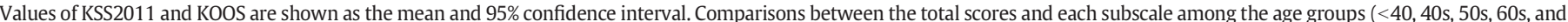

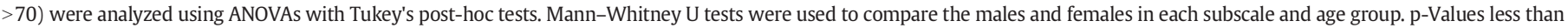
0.05 are shown as *: $\mathrm{p}<0.05$ vs $<40$. $\uparrow: \mathrm{p}<0.05$ vs 40 s. $\ddagger$ : $\mathrm{p}<0.05$ vs $50 \mathrm{~s}$. $\S$ : $\mathrm{p}<0.05$ vs $60 \mathrm{~s}$. $\mathrm{q}: \mathrm{p}<0.05$ between males and females.

ADL: activities of daily living; QOL: quality of life; overall KOOS: the total KOOS summed from the individual subscale scores. 
a

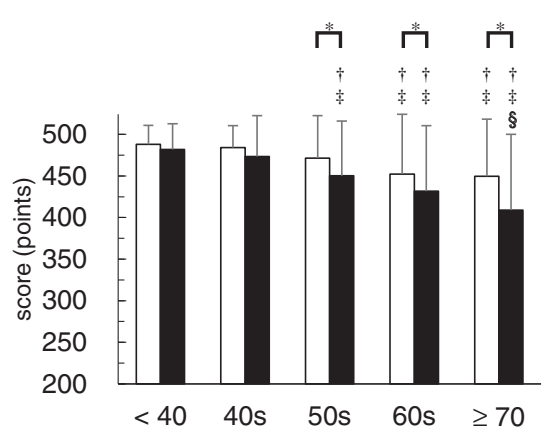

C

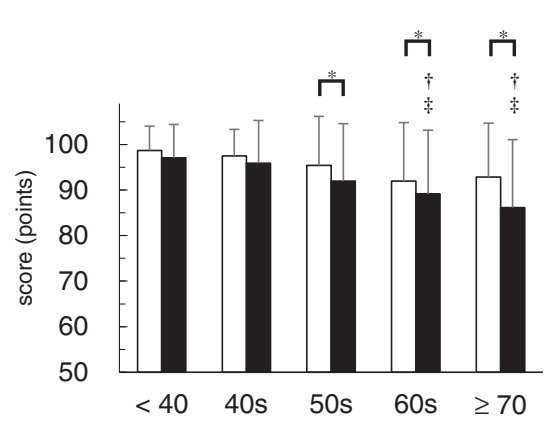

e

Sports/recreation

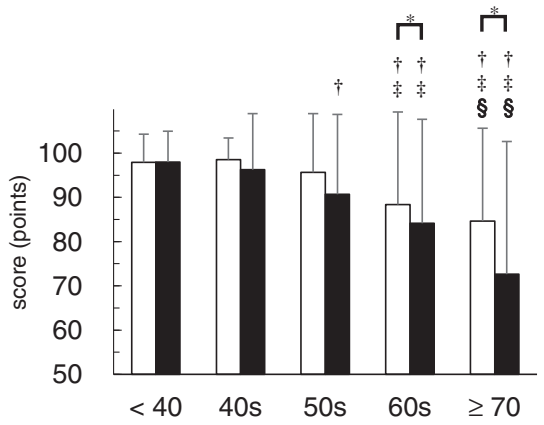

b Pain

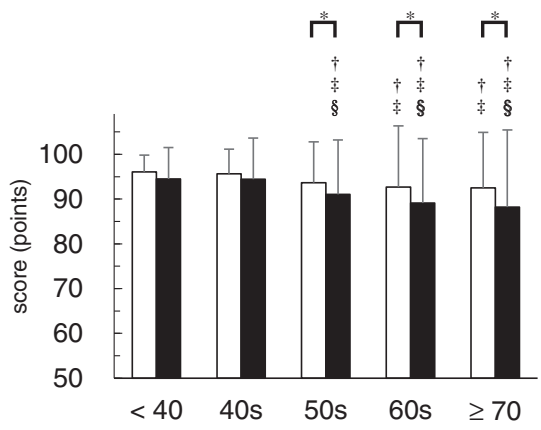

d

ADL

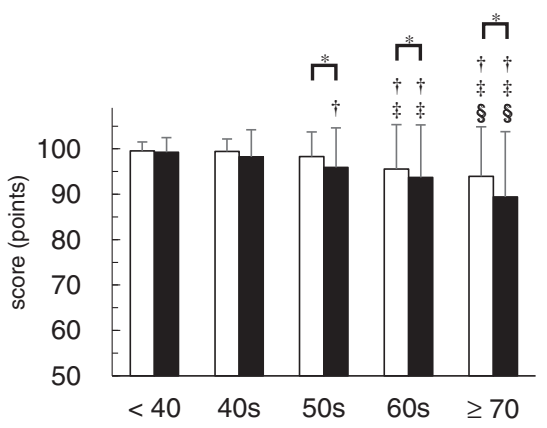

f

QOL

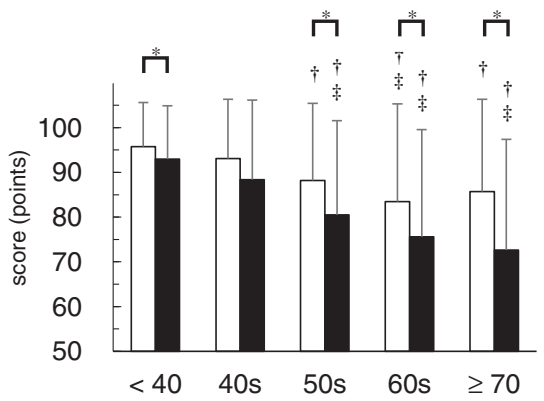

male female

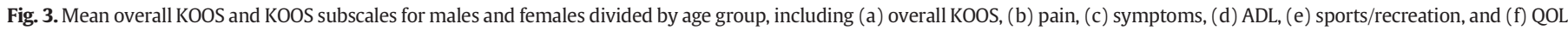

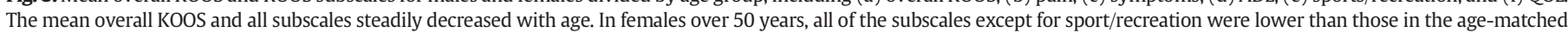

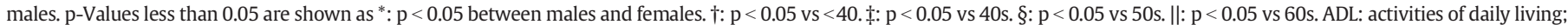
QOL: quality of life; Overall KOOS: the total KOOS summed from the individual subscales.

indicating better functional status, and the maximum score on each subscale was 100 points.

\subsection{Statistical analysis}

The participants were classified into five subgroups by age: under 40 years old, 40s, 50s, 60s, and over 70 years old, and by KL grades: 0 , 1, 2, 3, and 4. The mean KSS2011 and KOOS by age and KL grade were compared among each group using analyses of variance (ANOVAs) with Tukey's post-hoc tests. Mann-Whitney U tests were performed to evaluate differences between females and males within each score. Furthermore, to evaluate the association between the KL grade and both scores, multiple regression analysis was performed. The overall KSS2011 and overall KOOS were assumed to be dependent variables, and age, sex, BMI, and KL grade were assumed to be independent variables. All analyses were performed in SPSS ver. 22.0, and p-values less than 0.05 were considered significant.
3. Results

3.1. Prevalence of knee osteoarthritis

Data were obtained for all 963 participants, which included 368 male and 595 female volunteers with an average age of 54.7 years (range: 20 to 90 ). The participants who were more than 70 were the oldest age group in this study. Three-hundred-ninety-seven participants (40\%) showed radiographic knee OA. The prevalence of knee OA in females was higher than that in males in all age groups and increased with age (Table 1). Most of the participants who had knee OA were classified as KL grade 2 (Fig. 1).

\subsection{Age- and sex- related differences in KSS 2011}

The overall KSS2011 and each subscale tended to gradually decrease with age (Fig. 2a and Table 2). The score of overall KSS2011 went down by approximately 10 points at over 70 years old from under 40 years old in males and females: 140.9 . (95\% confidence interval (CI): 138.3 to 143.4 ), 136.7 (95\% CI: 132.4 to 141.1 ), 135.7 (95\% CI: 131.6 to 139.8 ), 130.3 (95\% CI: 126.3 to 134.3 ), 132.5 (95\% CI: 128.1 to 136.7 ) regarding males under 40 years old, in their 40s, 50s, 60s, and over 70 years old, and 141.5 (95\% CI: 139.2 to 143.7 ) 136.0 (95\% CI: 132.4 to 139.7 ), 132.1 (95\% CI: 129.2 to 135.0 ), 131.5 (95\% CI: 128.9 to 134.1 ), 128.4 (95\% CI: 124.5 to 132.2 ) regarding females. Except for the walking and standing scores in participants older than 70 years, most subscales did not show sex-related 
differences (Fig. 2b to f). The change of the symptoms score, standard activities score, and advanced activities score was less than 2 points by age group (Fig. $2 \mathrm{~b}, \mathrm{e}, \mathrm{f}$ ). The patient satisfaction score declined steadily from under 40 years old to over 70 years old in both sexes. (Fig. 2c and Table 2).

\subsection{Age- and sex-related differences in KOOS}

The mean overall KOOS and all subscales steadily decreased with age (Fig. 3a to f). The overall KOOS was significantly lower in females beginning in their 50 s and in males beginning in their 60s (Table 2). The ADL, QOL, and sports/recreation scores were clearly lower in participants in their 60s and older (Table 2 and Fig. 3d, e, f). In females older than 50 , all of the subscale scores except for sport/recreation were lower than the age-matched subscale scores in male participants.

\subsection{KL grade related differences in KSS2011 and KOOS}

The mean overall KSS2011 gradually decreased with KL grade (Table 3 and Fig. 4a): 137.8 (95\% CI: 135.4 to 140.2 ), 136.4 (95\% CI: 133.5 to 139.3 ), 129.5 (95\% CI: 124.1 to 135.0), 130.1 (95\% CI: 124.9 to 135.3 ), 126.2 (95\% CI: 103.3 to 149.1 ) regarding males in KL grade 0, KL grade 1, KL grade 2, KL grade 3, and KL grade 4, and 139.5 (95\% CI: 137.2 to 141.7 ), 134.9 (95\% CI: 133.0 to 137.6 ), 131.7 (95\% CI: 129.4 to 133.9 ), 129.0 (95\% CI: 125.0 to 133.0 ), 123.5 (95\% Cl: 110.2 to 136.9 ) regarding females. The symptoms, patient satisfaction score, and walking and standing score declined steadily from under KL grade 0 to KL grade 4 in both sexes (Fig. $4 \mathrm{~b}$ to d). The change of the standard activities score and advanced activities score was less than 3 points by KL grade (Fig. 4 e, f).

The mean overall KOOS and all its subscales were clearly decreased with increased KL grade (Table 3 and Fig. 5a to f). In particular, a distinct decrease was observed from KL grades 2 to 4 . In KL grade 3, there were sex-related differences in all components. The score of KL grade 4 was lower than those in the smaller grade groups for all components. The score of KL grade 4 was lower than those in the smaller grade groups for all components.

\subsection{Association with KL grade in KSS2011 and KOOS}

The overall KSS2011 was significantly correlated with age and KL grade. On the other hand, the overall KOOS was significantly correlated with sex, age, BMI, and KL grade (Table 4). The KL grade was more strongly associated with the KOOS ( $\beta=-0.42$, $\mathrm{p}<0.001)$ than with the KSS2011 $(\beta=-0.13, \mathrm{p}=0.001)$.
Table 4

Correlations of individual factors with the overall KOOS and KSS2011 assessed by multiple linear regression analysis.

\begin{tabular}{|c|c|c|c|c|c|c|c|c|}
\hline \multirow{3}{*}{ Factors } & \multicolumn{4}{|c|}{ Overall KOOS } & \multicolumn{4}{|c|}{ Overall KSS } \\
\hline & \multirow[t]{2}{*}{$\beta$} & \multicolumn{2}{|l|}{$95 \% \mathrm{CI}$} & \multirow[t]{2}{*}{ p-Value } & \multirow[t]{2}{*}{$\beta$} & \multicolumn{2}{|l|}{$95 \% \mathrm{CI}$} & \multirow[t]{2}{*}{ p-Value } \\
\hline & & lower & upper & & & lower & upper & \\
\hline Sex & -0.07 & -16.91 & -1.17 & 0.024 & 0.01 & -2.10 & 2.46 & 0.877 \\
\hline Age & -0.08 & -0.64 & -0.05 & 0.020 & -0.16 & -0.27 & -0.10 & $<0.001$ \\
\hline BMI & -0.14 & -3.99 & -1.75 & $<0.001$ & 0.03 & -0.15 & 0.49 & 0.306 \\
\hline KLG & -0.42 & -30.48 & -21.85 & $<0.001$ & -0.13 & -3.35 & -0.85 & 0.001 \\
\hline
\end{tabular}

The dependent variables were total KOOS and KSS2011, and the independent variables were sex, age, BMI, and KLG.

$\beta$ : regression coefficient; $95 \% \mathrm{CI}$ : 95\% confidence interval; BMI: body mass index; KLG: Kellgren-Lawrence grade.

\section{Discussion}

The aim of this study was to determine population-based reference data for the KSS2011 and KOOS and to correlate those scores with the severity of knee OA using large-scale epidemiological data from the general Japanese population. The overall KSS2011 decreased with age regardless of sex. Differences between the sexes were more distinct in the KOOS than in the KSS2011. In addition, radiographic knee OA was more strongly associated with the KOOS than the KSS2011. To our knowledge, this is the first study to evaluate knee complaints across the entire adult population as measured with both the KSS2011 and KOOS.

Knee function and physical activities vary among patients and are influenced by age and sex. As expected, the overall KSS2011 and KOOS decreased with age in the current study, as has been previously reported in other populations [19-21]. The results reported here are consistent with those previous findings. Almost none of the KSS2011 subscales showed differences by sex, and the decrease with age was subtle. In contrast, the KOOS significantly decreased with age, and there were

Table 3

KSS2011 and KOOS reported as mean and 95\% confidence interval for males (M) and females (F) by KL grade.

\begin{tabular}{|c|c|c|c|c|c|c|c|c|c|c|}
\hline & \multicolumn{2}{|l|}{ KL grade 0} & \multicolumn{2}{|l|}{ KL grade 1} & \multicolumn{2}{|l|}{ KL grade 2} & \multicolumn{2}{|l|}{ KL grade 3} & \multicolumn{2}{|l|}{ KL grade 4} \\
\hline & M & $\mathrm{F}$ & M & $\mathrm{F}$ & M & $\mathrm{F}$ & M & $\mathrm{F}$ & M & $\mathrm{F}$ \\
\hline \multirow[t]{3}{*}{ Overall KSS2011 } & 137.8 & 139.5 & 136.4 & 134.9 & $129.5^{*}$ & $131.7^{*}$ & 130.1 & $129.0^{*} \dagger$ & 126.2 & $123.5^{*}$ \\
\hline & 135.4 to & 137.2 to & 133.5 to & 132.3 to & 124.1 to & 129.4 to & 124.9 to & 125.0 to & 103.3 to & 110.2 to \\
\hline & 140.2 & 141.7 & 139.3 & 137.6 & 134.9 & 133.9 & 135.3 & 133.0 & 149.1 & 136.9 \\
\hline \multirow[t]{2}{*}{ Symptoms } & 23.5 & 23.8 & 23.5 & 23.1 & 22.5 & $22.5^{*}$ & 22.6 & $22.0^{*}$ & $17.4^{*}+\$ \S$ & $20.7^{*}$ \\
\hline & 23.1 to 24.0 & 23.4 to 24.2 & 22.9 to 24.1 & 22.5 to 23.7 & 21.5 to 23.5 & 21.9 to 23.1 & 21.0 to 24.2 & 21.2 to 22.8 & 11.2 to 236 . & 17.8 to 23.7 \\
\hline \multirow[t]{2}{*}{ Patient satisfaction } & 34.2 & 34.7 & 32.6 & 32.8 & 31.3 & $30.7^{*}$ & $29.4^{*}$ & $30.3^{*}$ & 30.0 & 30.0 \\
\hline & 33.1 to 35.3 & 33.5 to 35.9 & 31.1 to 34.0 & 31.6 to 34.0 & 29.2 to 33.5 & 29.6 to 31.7 & 26.5 to 32.2 & 28.7 to 32.0 & 20.2 to 39.8 & 25.6 to 34.4 \\
\hline \multirow{2}{*}{$\begin{array}{l}\text { Walking and } \\
\text { standing }\end{array}$} & 28.4 & 28.0 & 27.9 & 27.5 & $26.4^{*}$ & 27.4 & 26.9 & $26.1^{*}$ & 26.4 & 26.2 \\
\hline & 27.8 to 29.0 & 27.2 to 28.8 & 27.1 to 28.8 & 26.7 to 28.2 & 24.9 to 27.8 & 26.8 to 28.1 & 24.9 to 28.9 & 24.9 to 27.2 & 21.5 to 31.3 & 22.8 to 29.6 \\
\hline \multirow[t]{2}{*}{ Standard activities } & 28.7 & 29.2 & 29.1 & 28.6 & $27.5^{*} \dagger$ & 28.4 & 28.6 & 28.2 & 28.6 & $26.9 * \dagger$ \\
\hline & 28.3 to 29.2 & 28.8 to 29.6 & 28.6 to 29.5 & 28.1 to 29.2 & 26.4 to 28.5 & 28.0 to 28.9 & 27.8 to 29.4 & 27.4 to 29.0 & 24.7 to 32.5 & 24.3 to 29.4 \\
\hline \multirow[t]{2}{*}{ Advanced activities } & 23.0 & 23.7 & 23.4 & 23.0 & 21.8 & 22.6 & 22.6 & 22.3 & 23.8 & 19.7 \\
\hline & 22.4 to 23.6 & 23.2 to 24.2 & 22.7 to 24.0 & 22.3 to 23.6 & 20.5 to 23.2 & 22.0 to 23.2 & 21.2 to 23.9 & 21.3 to 23.4 & 20.5 to 27.1 & 15.6 to 23.9 \\
\hline \multirow[t]{2}{*}{ Overall KOOS } & 486.9 & 483.3 & $468.3^{*}$ & 471.0 & $458.6^{*} \mathrm{~g}$ & $448.9 * \dagger$ & $422.1^{*}+9$ & $379.8 * 1 \ddagger$ & $285.4^{*} \dagger \leftrightarrows \S$ & $275.1^{*}+\varsigma$ \\
\hline & 483.5 to 490.3 & 478.4 to 488.2 & 458.7 to 477.9 & 464.0 to 478.0 & 443.7 to 473.4 & 440.7 to 457.1 & 386.4 to 457.8 & 361.2 to 398.3 & 211.5 to 359.3 & 217.9 to 332.3 \\
\hline \multirow[t]{2}{*}{ Pain } & 98.5 & 97.8 & $95.1^{*}$ & 95.8 & $93.1^{*}$ & $91.9^{*} \dagger$ & $88.1^{*} \dagger 9$ & $80.6^{*} \dagger$ & $58.9 *+7 \S$ & $61.7^{*}+\S$ \\
\hline & 97.9 to 99.1 & 96.9 to 98.8 & 93.3 to 96.9 & 94.5 to 97.0 & 90.4 to 95.8 & 90.3 to 93.5 & 81.6 to 94.6 & 77.1 to 84.0 & 44.8 to 73.0 & 48.4 to 75.0 \\
\hline \multirow[t]{2}{*}{ Symptoms } & 96.5 & 95.1 & 94.2 & 94.7 & $92.7^{*}$ & 91.9 & $88.9 * \dagger 9$ & $82.5^{*}+$ & $62.1^{*}+\$$ & $61.4 * \dagger \S$ \\
\hline & 95.8 to 97.3 & 94.0 to 96.2 & 92.3 to 96.1 & 93.5 to 96.0 & 89.9 to 95.5 & 90.4 to 93.5 & 82.9 to 95.0 & 79.3 to 85.7 & 51.0 to 73.3 & 49.3 to 73.5 \\
\hline \multirow[t]{2}{*}{ ADL } & 99.4 & 99.4 & 97.5 & 97.7 & $96.5^{*}$ & $96.1^{*}$ & $90.8^{*}+\frac{9}{9}$ & $86.9^{*} \dagger$ & $74.1^{*} \dagger \S$ & $69.6 *+\div \S$ \\
\hline & 99.2 to 99.7 & 99.0 to 99.7 & 96.3 to 98.7 & 96.8 to 98.6 & 94.6 to 98.4 & 95.0 to 97.3 & 84.9 to 96.6 & 83.9 to 89.9 & 54.9 to 93.3 & 58.7 to 80.5 \\
\hline \multirow[t]{2}{*}{ Sports/recreation } & 97.7 & 98.2 & 93.0 & $94.5^{*}$ & $90.8^{*} \dagger$ & 89.1 & $78.6^{*}+9$ & $67.9 * \dagger$ & $44.0 *+\ddagger \S$ & $38.7^{*}+\ddagger \S$ \\
\hline & 96.6 to 98.8 & 97.0 to 99.4 & 90.1 to 95.8 & 92.6 to 96.4 & 86.5 to 95.1 & 86.5 to 91.7 & 68.3 to 89.0 & 62.0 to 73.9 & 20.2 to 67.8 & 18.7 to 58.6 \\
\hline \multirow[t]{2}{*}{ QOL } & 94.8 & 92.8 & 88.5 & $88.3^{*}$ & $85.4^{*} \dagger 9$ & $79.9 * \dagger$ & 75.69 & $61.8 *+$ & $46.3^{*}+\S$ & $43.8 *+\$ \S$ \\
\hline & 93.0 to 96.5 & 90.6 to 95.0 & 85.4 to 91.7 & 85.6 to 90.9 & 80.5 to 90.4 & 77.2 to 82.5 & 65.4 to 85.9 & 56.4 to 67.2 & 26.0 to 66.5 & 31.5 to 56.0 \\
\hline
\end{tabular}

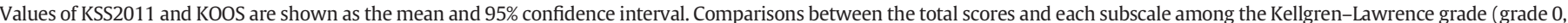

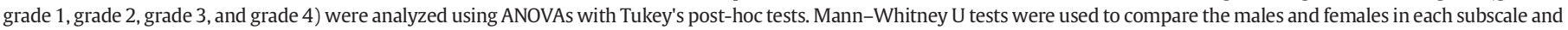

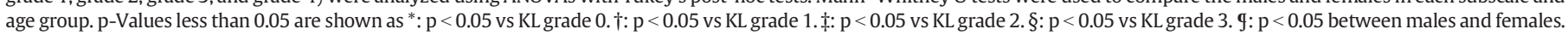
ADL: activities of daily living; QOL: quality of life; overall KOOS: the total KOOS summed from the individual subscale scores. 
a

Overall KSS2011

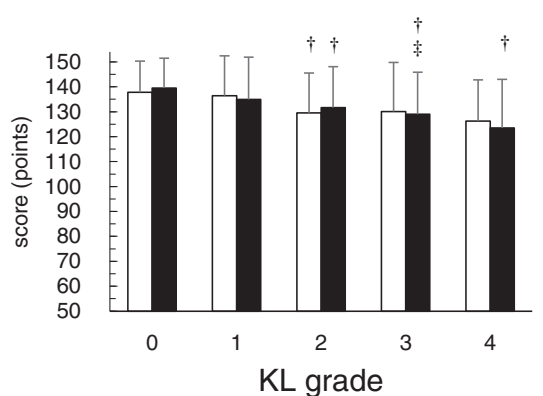

C

Patient satisfaction

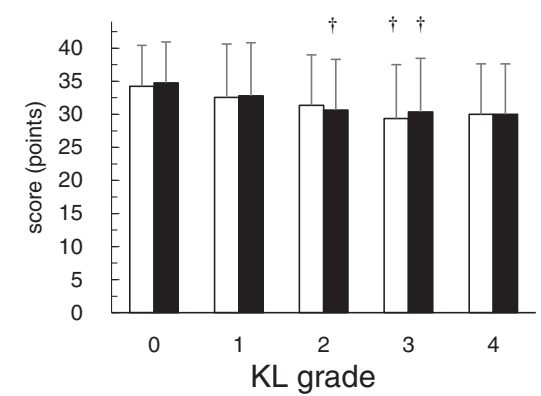

e

Standard activities

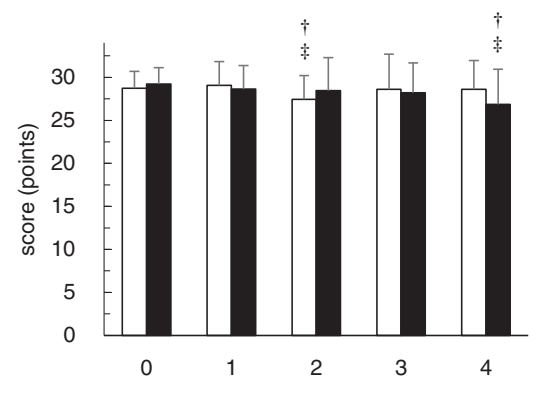

$\mathrm{KL}$ grade b Symptoms

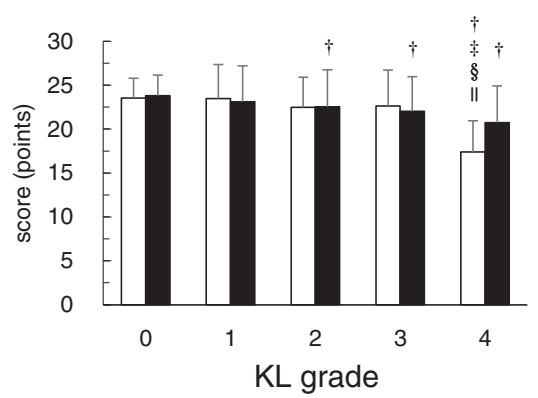

d Walking and standing

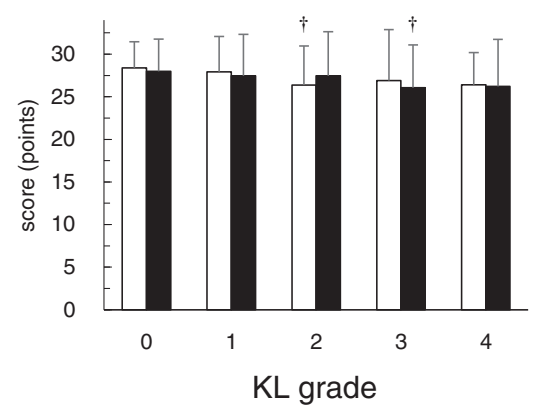

f Advanced activities

\section{$\square$ male $\square$ female}

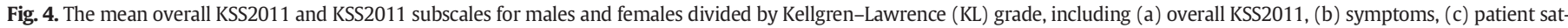

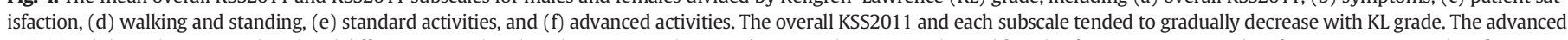

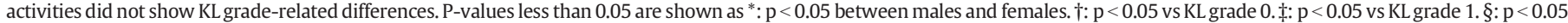
vs KL grade 2. \|: $\mathrm{p}<0.05$ vs KL grade 3. Overall KSS: the total KSS2011 summed from the individual subscales.

clear differences between male and female participants. Elderly females reported more knee-related complaints in all of the KOOS subscales than the age-matched males in this study cohort. Therefore, these characteristics of the KSS2011 and KOOS should be considered when evaluating patients. Furthermore, elderly patients typically set lower goals after knee operations than younger patients.

It is important to understand the factors that influence patientreported outcome scales. In the present study, the KSS2011 was significantly correlated with age and KL grade, and the KOOS was correlated with age, BMI, and KL grade. In particular, the KL grade was more strongly correlated with the KOOS than the KSS2011. A few previous studies have examined the association of the KSS2011 with factors in general populations. One prior cross-sectional study in Japan reported that the KSS2011 decreased with age and was associated with the KL glade, BMI, leg muscle strength, and the Timed Up and Go test [20]. Population-based normative data for the KOOS were reported and stratified by age and sex [21]. A previous study found that lower knee extension strength and higher BMI were associated with knee pain [4]. The relationship between radiographic knee OA and quadriceps strength has also been reported [22]. In that study, the authors found that quadriceps muscle strength decreased in patients after age 50 years and a tendency for muscle strength to decrease with the progression of knee $\mathrm{OA}$ grade between grades 0 and 1 in both males and females. The KOOS may more accurately reflect knee muscular strength because we found here that the functional subscales of the KOOS were more greatly decreased with age than those of the KSS2011 in patients older than 50 years. From a clinical perspective, the KOOS may be more useful than the KSS2011 to assess knee complaints from the initial phase to the late phase of radiographic knee OA.

There were several limitations to this study. First, we cannot completely exclude the effects of other systemic diseases. Any physical function-related comorbidity could affect the results. Second, this study was performed in a limited local region, which may not be representative of Japan overall. For example in the Research on Osteoarthritis-osteoporosis Against Disability (ROAD) study, living in a mountainous community was a risk factor for knee OA $[23,24]$. Third, we used the Japanese version of the KSS2011, for which validation 

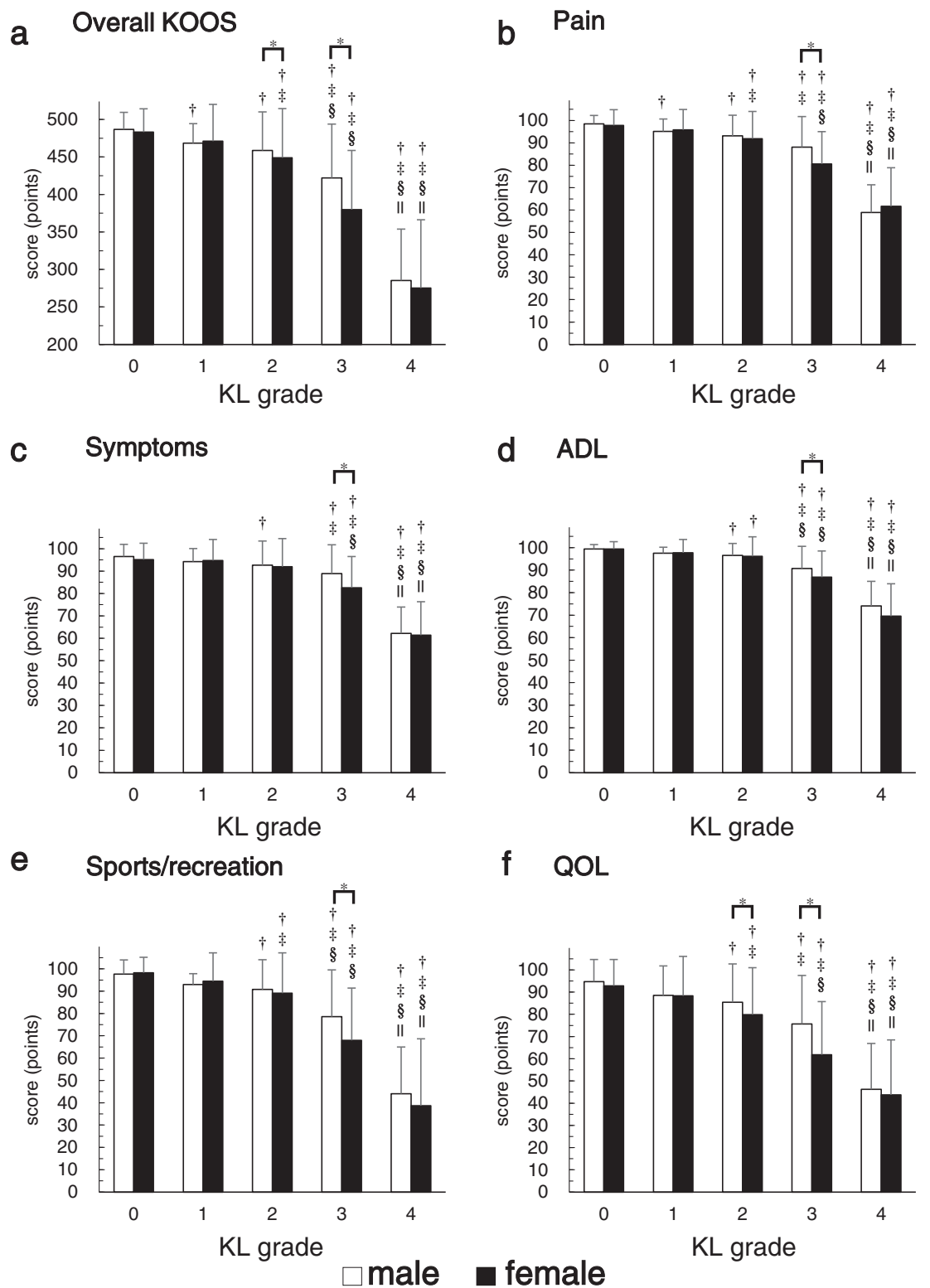

f QOL

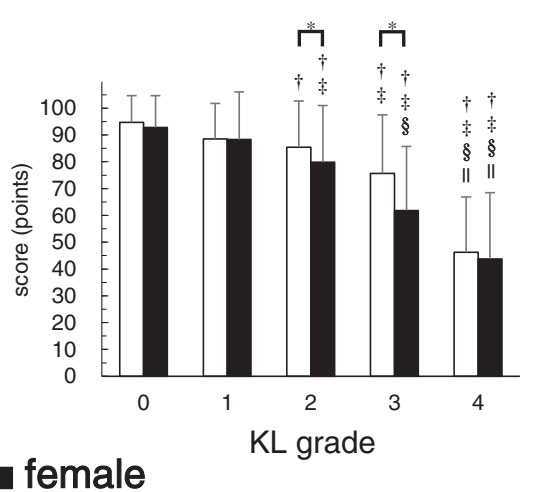

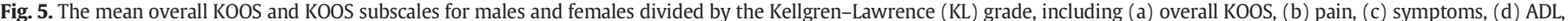

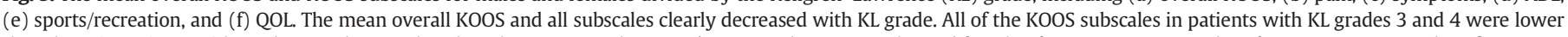

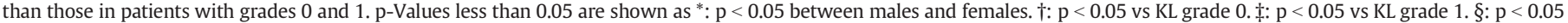
vs KL grade 2. $\|: \mathrm{p}<0.05$ vs KL grade 3. ADL: activities of daily living; QOL: quality of life; Overall KOOS: the total KOOS summed from the individual subscales.

work using cultural adaptation methods has not yet been performed. For populations with larger cultural differences from Western countries, such as Asian populations, the cultural adaptation may be important [9]. Finally, smaller differences in the results than anticipated may have been caused by selection bias resulting from the use of voluntary participants who may be more health conscientious than the population overall.

\section{Conclusion}

This is the first study to evaluate knee complaints as measured by both the KSS2011 and KOOS in a large general population in Japan. The three key findings of the present study are: (1) the KSS2011 and KOOS decreased with age and were correlated with the KL grade. (2) Sex differences were more apparent in the KOOS than in the KSS2011. (3) The KOOS was more strongly correlated with the KL grade than KSS2011 across the entire adult population. In the general population, the KOOS may be more useful than the KSS2011 when evaluating knee complaints in patients with knee OA.

\section{Conflict of interest statement}

No benefits in any form have been received or will be received from any commercial party related directly or indirectly to the subject of this article entitled "The Knee injury and Osteoarthritis Outcome Score reflects the severity of knee osteoarthritis better than the revised Knee Society Score in a general Japanese population".

\section{Acknowledgments}

This study was supported in part by a Grant-in-Aid from the Ministry of Education, Culture, Sports, Science and Technology of Japan (No. 18200044), the Karoji Memorial Fund for Medical Research [No. 5310139302], the Japanese Society for the Promotion of Science (No. 
21500676), and a JOA Subsidized Science Project Research from the Japanese Orthopaedic Association (5310120126).

\section{References}

[1] Silverwood V, Blagojevic-Bucknall M, Jinks C, Jordan JL, Protheroe J, Jordan KP. Current evidence on risk factors for knee osteoarthritis in older adults: a systematic review and meta-analysis. Osteoarthritis Cartilage 2015;23:507-15.

[2] Anwer S, Alghadir A. Effect of isometric quadriceps exercise on muscle strength, pain, and function in patients with knee osteoarthritis: a randomized controlled study. J Phys Ther Sci 2014;26:745-8.

[3] Matsumoto T, Hashimura M, Takayama K, Ishida K, Kawakami Y, Matsuzaki T, et al. A radiographic analysis of alignment of the lower extremities-initiation and progression of varus-type knee osteoarthritis. Osteoarthritis Cartilage 2015;23:217-23.

[4] Zhai G, Blizzard L, Srikanth V, Ding C, Cooley H, Cicuttini F, et al. Correlates of knee pain in older adults: Tasmanian Older Adult Cohort Study. Arthritis Rheum 2006; 55:264-71.

[5] Kurtz S, Ong K, Lau E, Mowat F, Halpern M. Projections of primary and revision hip and knee arthroplasty in the United States from 2005 to 2030. J Bone Joint Surg Am 2007;89:780-5.

[6] Collins NJ, Misra D, Felson DT, Crossley KM, Roos EM. Measures of knee function: International Knee Documentation Committee (IKDC) Subjective Knee Evaluation Form, Knee Injury and Osteoarthritis Outcome Score (KOOS), Knee Injury and Osteoarthritis Outcome Score Physical Function Short Form (KOOS-PS), Knee Outcome Survey Activities of Daily Living Scale (KOS-ADL), Lysholm Knee Scoring Scale, Oxford Knee Score (OKS), Western Ontario and McMaster Universities Osteoarthritis Index (WOMAC), Activity Rating Scale (ARS), and Tegner Activity Score (TAS). Arthritis Care Res 2011;63(Suppl. 11):S208-28.

[7] Insall JN, Dorr LD, Scott RD, Scott WN. Rationale of the Knee Society clinical rating system. Clin Orthop Relat Res 1989:13-4.

[8] Scuderi GR, Bourne RB, Noble PC, Benjamin JB, Lonner JH, Scott WN. The new Knee Society Knee Scoring System. Clin Orthop Relat Res 2012;470:3-19.

[9] Dinjens RN, Senden R, Heyligers IC, Grimm B. Clinimetric quality of the new 2011 Knee Society score: high validity, low completion rate. Knee 2014;21:647-54.

[10] Debette C. French adaptation of the new Knee Society Scoring System for total knee arthroplasty. Orthop Traumatol Surg Res 2014;100:531-4.

[11] Noble PC, Scuderi GR, Brekke AC, Sikorskii A, Benjamin JB, Lonner JH, et al. Development of a new Knee Society scoring system. Clin Orthop Relat Res 2012;470:20-32.
[12] Nakamura N, Takeuchi R, Sawaguchi T, Ishikawa H, Saito T, Goldhahn S. Crosscultural adaptation and validation of the Japanese Knee Injury and Osteoarthritis Outcome Score (KOOS). J Orthop Sci 2011;16:516-23.

[13] Sasaki E, Tsuda E, Yamamoto Y, Meada S, Otsuka H, Ishibashi Y. Relationship between patient-based outcome score and conventional objective outcome scales in post-operative total knee arthroplasty patients. Int Orthop 2014;38:373-8.

[14] Matsuda S, Kawahara S, Okazaki K, Tashiro Y, Iwamoto Y. Postoperative alignment and ROM affect patient satisfaction after TKA. Clin Orthop Relat Res 2013;471: 127-33.

[15] Inoue R, Ishibashi Y, Tsuda E, Yamamoto Y, Matsuzaka M, Takahashi I, et al. Knee osteoarthritis, knee joint pain and aging in relation to increasing serum hyaluronan level in the Japanese population. Osteoarthritis Cartilage 2011;19:51-7.

[16] Sasaki E, Ishibashi Y, Tsuda E, Ono A, Yamamoto Y, Inoue R, et al. Evaluation of locomotive disability using loco-check: a cross-sectional study in the Japanese general population. J Orthop Sci 2013;18:121-9.

[17] Kellgren JH, Lawrence JS. Radiological assessment of osteo-arthrosis. Ann Rheum Dis 1957;16:494-502

[18] Roos EM, Roos HP, Lohmander LS, Ekdahl C, Beynnon BD. Knee Injury and Osteoarthritis Outcome Score (KOOS)-development of a self-administered outcome measure. J Orthop Sports Phys Ther 1998;28:88-96.

[19] Badley EM, Tennant A. Changing profile of joint disorders with age: findings from a postal survey of the population of Calderdale, West Yorkshire, United Kingdom. Ann Rheum Dis 1992;51:366-71.

[20] Taniguchi N, Matsuda S, Kawaguchi T, Tabara Y, Ikezoe T, Tsuboyama T, et al. The K.S.S. 2011 reflects symptoms, physical activities, and radiographic grades in a Japanese population. Clin Orthop Relat Res 2015;473:70-5.

[21] Paradowski PT, Bergman S, Sunden-Lundius A, Lohmander LS, Roos EM. Knee complaints vary with age and gender in the adult population. Population-based reference data for the Knee injury and Osteoarthritis Outcome Score (KOOS). BMC Musculoskelet Disord 2006;7:38.

[22] Omori G, Koga Y, Tanaka M, Nawata A, Watanabe H, Narumi K, et al. Quadriceps muscle strength and its relationship to radiographic knee osteoarthritis in Japanese elderly. J Orthop Sci 2013;18:536-42.

[23] Muraki S, Oka H, Akune T, Mabuchi A, En-yo Y, Yoshida M, et al. Prevalence of radiographic knee osteoarthritis and its association with knee pain in the elderly of Japanese population-based cohorts: the ROAD study. Osteoarthritis Cartilage 2009; 17:1137-43.

[24] Muraki S, Akune T, Nagata K, Ishimoto Y, Yoshida M, Tokimura F, et al. Does osteophytosis at the knee predict health-related quality of life decline? A 3-year follow-up of the ROAD study. Clin Rheumatol 2015;34:1589-97. 